\title{
A44 POLYMORPHISMS IN THE INTERLEUKIN 4, INTERLEUKIN 13 AND CORRESPONDING RECEPTOR GENES ARE NOT ASSOCIATED WITH SYSTEMIC SCLEROSIS AND DO NOT INFLUENCE GENE EXPRESSION
}

JCA Broen, ${ }^{1}$ PDieude, ${ }^{2}$ MC Vonk, ${ }^{1}$ LBeretta, ${ }^{3}$ B Rueda, ${ }^{4}$ A Herrick, ${ }^{5}$ J Worthington, ${ }^{5}$ N Hunzelmann, ${ }^{6}$ G Riemekasten, ${ }^{7}$ H Kiener, ${ }^{8}$ R Scorza, ${ }^{3}$ C P Simeon, ${ }^{9}$ V Fonollosa ${ }^{9}$ (for the Spanish Systemic Sclerosis group), P Carreira, ${ }^{10} \mathrm{~N}$ Ortego-Centeno, ${ }^{11}$ M A Gonzalez-Gay, ${ }^{12}$ P Airo, ${ }^{13}$ M J H Coenen, ${ }^{14}$ A Aliprantis, ${ }^{15} \mathrm{~J}$ Martin, ${ }^{4}$ Y Allanore, ${ }^{16,17}$ T R D J Radstake ${ }^{1}$ Deparment of Rheumatology, Radboud University Nijmegen Medical Center, Nijmegen, The Netherlands; 2 Service de Rhumatologie, Université Diderot Paris 7, Paris, France; ${ }^{3}$ Referral Center for Systemic Autoimmune Diseases, University of Milan, Milan, Italy; ${ }^{4}$ Instituto de Parasitología y Biomedicina, CSIC, Granada, Spain; ${ }^{5}$ Rheumatic Diseases Centre, University of Manchester, Salford Royal NHS Foundation Trust, Manchester, UK; ${ }^{6}$ Department of Dermatology, University of Cologne, Cologne, Germany; ${ }^{7}$ Department of Rheumatology and Clinical Immunology, Charité University Hospital and German Rheumatism Research Centre, a Leibniz institute, Berlin, Germany; ${ }^{8}$ Department of Internal Medicine, Division of Rheumatology, University of Vienna, Vienna, Austria; ${ }^{9}$ Servicio de Medicina Interna, Hospital Vall d'Hebron, Barcelona, Spain; ${ }^{10}$ Servicio de Reumatologia, Hospital 12 de Octubre, Madrid, Spain; ${ }^{11}$ Servicio de Medicina Interna, Hospital Xeral-Calde, Lugo, Spain; ${ }^{12}$ Servicio de Reumatologia, Hospital Marques de Valdecillas, Santander, Spain; ${ }^{13}$ Servizio di Reumatologia ed Immunologia Clinica, Spedali Civili, Brescia, Italy; ${ }^{14}$ Department of Human Genetics, Radboud University Nijmegen Medical Center, Nijmegen, The Netherlands; ${ }^{15}$ Department of Immunology and Infectious Diseases, Harvard School of Public Health, Boston, Massachusetts, USA; ${ }^{16}$ INSERM U781, Université Paris Descartes, Hôpital Necker, Paris, France; ${ }^{17}$ Service de Rhumatologie A, Université Paris Descartes, Hôpital Cochin, Paris, France

JM, YA and TRDJR contributed equally to this work

10.1136/ard.2010.148965.14

Aim Polymorphisms in the interleukin 4 (IL-4), IL-13 and their corresponding receptors have previously been found associated with systemic sclerosis (SSc). In this study the authors aim to validate these previous observations and scrutinise their effects on gene expression.

Patients and methods The authors genotyped a cohort consisting of 2488 SSc patients and 2246 healthy controls, derived from The Netherlands, Spain, UK, Italy, Germany 
and France. Taqman assays were used for genotyping two single-nucleotide polymorphisms (SNPs) within IL-4 (Q576R/ $\mathrm{rs} 1801275)$ and the $I L-4 \alpha$ receptor $(-590 \mathrm{C} / T / \mathrm{rs} 2243250)$. In the IL-13 gene two SNPs were genotyped R130Q (rs20541) and $-1112 C / T$ (rs1800925.) In the $I L-13 \alpha$ receptor gene, the 43163:G/A (rs6646259) variant was genotyped. In addition, the authors investigated the effect of these polymorphisms on corresponding gene expression with RT-PCR in B cells, T cells, plasmacytoid dendritic cells, monocytes and myeloid dendritic cells.

Results None of these polymorphisms was found to be enriched in the SSc population or in any SSc clinical subtype and there was no influence of these polymorphisms on development of either pulmonary arterial hypertension and declineer of forced vital capacity in 15 years of follow-up. In addition, the authors did not observe an effect on expression levels in the cell subtypes.

Conclusions This data show that these polymorphisms do not play a role in SSc and do not influence gene expression levels. 